\title{
Carbon nanohybrids used as catalysts and emulsifiers for reactions in biphasic aqueous/organic systems
}

\author{
Daniel E. Resasco* \\ School of Chemical, Biological and Materials Engineering, and Center for Interfacial Reaction Engineering (CIRE), University of Oklahoma, Norman, OK, \\ 73019, USA
}

A R T I C L E I N F O

Article history:

Received April 172014

Accepted April 272014

Published 20 June 2014

Keywords:

Carbon nanomaterials

Nanohybrids

Emulsions

Biphasic aqueous/organic mixtures

Catalysis at water/oil interface

Biomass conversion

Fischer-Tropsch synthesis

\begin{abstract}
A B S T R A C T
This mini-review summarizes some novel aspects of reactions conducted in aqueous/organic emulsions stabilized by carbon nanohybrids functionalized with catalytic species. Carbon nanohybrids represent a family of solid catalysts that not only can stabilize water-oil emulsions in the same fashion as Pickering emulsions, but also catalyze reactions at the liquid/liquid interface. Several examples are discussed in this mini-review. They include (a) aldol condensation-hydrodeoxygenation tandem reactions catalyzed by basic (MgO) and metal (Pd) catalysts, respectively; (b) FischerTropsch synthesis catalyzed by carbon-nanotube-supported $\mathrm{Ru}$; and (c) emulsion polymerization of styrene for the production of conductive polymer composites. Conducting these reactions in emulsion generates important advantages, such as increased liquid/liquid interfacial area that consequently means faster mass transfer rates of molecules between the two phases, effective separation of products from the reaction mixture by differences in the water-oil solubility, and significant changes in product selectivity that can be adjusted by modifying the emulsion characteristics.
\end{abstract}

(C) 2014, Dalian Institute of Chemical Physics, Chinese Academy of Sciences. Published by Elsevier B.V. All rights reserved.

\section{Introduction}

Reactions in biphasic aqueous/organic mixtures are potentially interesting for industrial processes, in which the use of two liquid phases is unavoidable due to the feedstock composition or when it provides an operational advantage. For example, in the upgrading of bio-fuels derived from biomass pyrolysis, phase separation and biphasic mixtures are unavoidable due to the high water concentration of the feedstock $(30 \%-40 \%)$ and the organic and less polar characteristics of the desirable product (deoxygenated fuels) [1]. In other cases, biphasic systems have been purposely created to facilitate the separation of products from unreacted reactants and catalysts, which can be recycled for maximum product yield [2].

Using emulsions in systems like these are attractive when valuable products can be separated into the other phase, preventing undesired secondary reactions. Partitioning of byproducts on the basis of their relative solubility can result in substantial simplifications in the separation stages, obviating the need for steps such as distillation that might damage heatsensitive compounds [3]. Moreover, emulsions greatly increase the liquid/liquid interfacial area, thus enhancing the rate of interfacial mass transfer. Molecular emulsifiers, such as detergents, polymers are typically employed to stabilize emulsions. Their role is to lower the interfacial tension between two phases, or create a film around the droplets making them repel each other, or increasing the viscosity of the continuous medium, maintain the droplets suspended. A major drawback of using molecular emulsifiers is their difficult separation from the reaction mixture, which requires expensive purification

\footnotetext{
*Corresponding author. Tel: +1-405-325-4370; Fax: +1-405-325-5813; E-mail: resasco@ou.edu This work was supported by the US Department of Energy/EPSCOR (DE SC00044136.85). 
methods, such as membrane filtration [4]. This disadvantage can be overcome by replacing the molecular surfactants with amphiphilic catalyst particles that can be readily recovered and reused after each reaction cycle [5]. This novel methodology combines the advantages of phase transfer and heterogeneous catalysis [6,7]: (a) increased interfacial area, (b) enhanced mass transfer of molecules between the two phases, (c) simplified reaction/separation process by using a recoverable solid catalyst instead of surfactant, and (d) effective separation of products from the reaction mixture by differences in the water-oil solubility (avoiding heating that leads to product decomposition).

Such process improvements could have a major impact in the field of biomass conversion to fuels [8-12] (upgrading of pyrolysis oil and sugars), production of specialty chemicals [13] pharmaceuticals, deep desulfurization of fuel oil [14,15], and Fischer-Tropsch synthesis (FTS) [16,17].

Furthermore, when these particles have a compartmentalized surface that displays different wettabilities (i.e., Janus particles), the stabilization of the emulsions becomes more effective [18-25]. Contrary to homogeneous particles that have uniform wettability and a fixed contact angle, the Janus particles are a more complex system with asymmetric wettability that can exhibit a tailored hydrophilic/hydrophobic balance, and therefore modify the location of the three-phase contact angle. The tunable surface properties of Janus nanoparticles have attracted attention for applications as diverse as sensing, electronics, photonics, and drug delivery [26-31].

In this contribution, a special case of Janus particles is discussed. They are the so-called carbon nanohybrids, which are composed of carbon nanomaterials (hydrophobic) fused to oxide nanoparticles (hydrophilic), which are able to stabilize emulsions and, when properly functionalized, catalyze reactions at the water/oil interface (i.e., liquid/solid/liquid interfacial catalysis) [5,32]. The amphiphilic character of these nanohybrids makes them segregate naturally to the water/oil interface, thus becoming suitable for the stabilization of emulsions with small droplet sizes with remarkable stability. Two important advantages of these nanohybrids over conventional Janus particles are a higher surface area and a higher extent of penetration into the organic liquid phase, due to the presence of the lipophilic carbon nanotubes. In addition, by using carbon nanotubes (CNT) it is possible to reduce the external diffusion limitations by functionalizing the outer surface of the CNT with the catalytic species. Nanotubes are more accessible than conventional supports having meso and microporosities. Additionally, by changing the type of CNT used one can vary the metal dispersion, e.g. multi-wall carbon nanotubes (MWCNT) have a more defective surface than single-wall carbon nanotubes (SWCNT) [33].

\section{Carbon nanohybrids as effective stabilizers of Pickering emulsions}

According to the Bancroft's rule [34], the continuous phase of an emulsion is that with a higher affinity for the emulsifier. The relative affinity of a given emulsifier for the two phases can be described in terms of the hydrophilic-lipophilic balance (HLB) [35]. When the emulsifier is a surfactant, the HLB number can be used to determine its ability to stabilize water-in-oil (low HLB, more hydrophobic) or oil-in-water (high HLB, more hydrophilic). In Pickering emulsions, fine solid particles adsorbed at the water/oil interface sterically hinder the coalescence of droplets and effectively stabilize the emulsion [36]. As shown in Fig. 1, when the contact angle between the water and the particle at the water/oil interface is $90^{\circ}$ the particle's tendency to stay at the interface is highest and the emulsion is most stable. Analogous to the case of surfactants, the phase with the highest affinity for the particle, i.e. the one that wets the particle more effectively will form the continuous phase. Therefore, a more hydrophilic particle will create a contact angle below $90^{\circ}$ and will tend to stabilize oil-in-water emulsions, while contrarily, a more hydrophobic particle will stabilize water-in-oil emulsions. Completely hydrophilic (or hydrophobic) particles will show the tendency to be solely in one of the phases and therefore the emulsions that they form are less stable. These trends can be changed when the water/oil volume ratio during the preparation of the emulsion is varied. For example, by increasing the amount of the dispersed phase, one can obtain the inverse emulsion $[19,24,32]$. That is, for a more hydrophobic particle that tends to form a water-in-oil emulsion, the inverse emulsion can be obtained at high water/oil volume ratios.

\subsection{SWCNTs carbon nanotubes/oxide nanoparticles}

An efficient nanohybrid for catalytic applications is one that has high stability at the water/oil interface, but at the same time, stabilizes catalytically active nanoparticles at the desired side of the interface. That is, one can expect that particles located on the hydrophobic end of the nanohybrid will catalyze reactions occurring in the organic phase, while those placed on the hydrophilic side will catalyze reactions in the aqueous phase [5]. In this sense, carbon nanohybrids are particularly attractive because they exhibit tunable properties that can be adjusted during synthesis or with simple post-synthesis treatments. For example, as produced pristine SWCNT are highly hydrophobic, but can be amphiphilic if they are combined with oxide nanoparticles. When the SWCNT are produced catalytically by decomposition of carbon-containing molecules (e.g., CO, methane, ethanol) over supported metal catalysts, one can

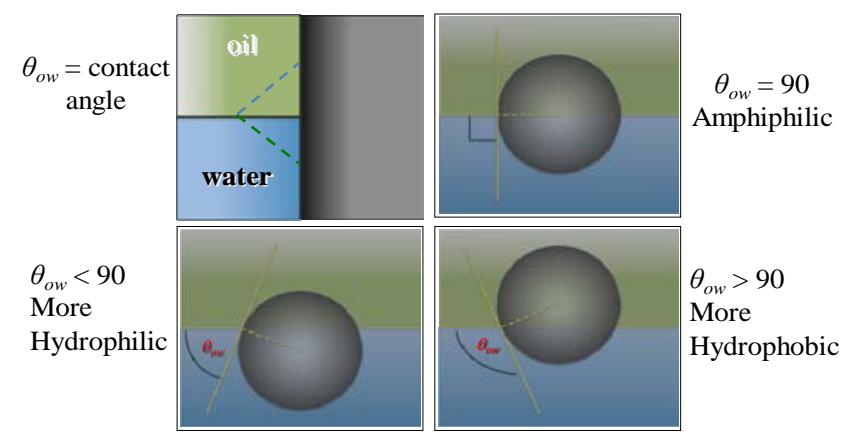

Fig. 1. Effect of the hydrophobic/hydrophilic balance on the contact angle of the water/oil interface and preferential position of the particle. 
leave the oxide support attached to the nanotubes. Typical supports for catalysts that are active and selective for the growth of SWCNT include $\mathrm{SiO}_{2}, \mathrm{MgO}, \mathrm{TiO}_{2}$, etc. [32]. As discussed below, these oxides can act as effective supports for catalytically active species (e.g. $\mathrm{Pd} / \mathrm{SiO}_{2}$ ) or as catalysts themselves (e.g. MgO). As illustrated in Fig. 2, by varying the SWCNT/silica ratio one can control the hydrophilic/hydrophobic balance and, therefore, tailor the characteristics of the resulting emulsion.

One of the various synthesis methods available for production of SWCNT in large scale is the CoMoCAT process [37], which has been operating industrially for several years [38]. In this process, SWCNT are obtained by CO disproportionation on supported Co-Mo catalysts. The support can be varied according to the desired nanotube characteristics (diameter, chirality, etc.) or in the case of nanohybrid production, according to the characteristics desired on the oxide nanoparticles (acidity/basicity, surface area, etc.). The synthesis is carried out on a fluidized bed reactor in above $650{ }^{\circ} \mathrm{C}$ and at moderate pressures $(<10 \mathrm{~atm})$ [37].

In the as-produced pristine SWCNT/silica nanohybrids, the hydrophobic fraction (SWCNT) becomes dominant with $\mathrm{C}$ contents as low as $4 \mathrm{wt} \%$. Therefore, the majority of the solid particles are wetted by oil. As a result, the interface bends away from the oil and concave towards the water, making oil the continuous phase, as illustrated in Fig. 3 [32].

\subsection{Functionalized carbon nanomaterials}

Oxidative functionalization greatly increases the hydrophilicity of SWCNTs $[39,40]$. Therefore, in addition to combining SWCNT and oxide nanoparticles at varying ratios to adjust the hydrophobic/hydrophilic balance, one can also increase hydrophilicity of the carbon materials by increasing the density of functional groups, such as $\mathrm{COOH}$. These functional groups $(-\mathrm{COOH})$ can be grafted on wall defects and ends of SWCNT by using an oxidant such as nitric acid [41,42] or plasma [43] treatments.

For example, by controlling the extent of SWCNT function-

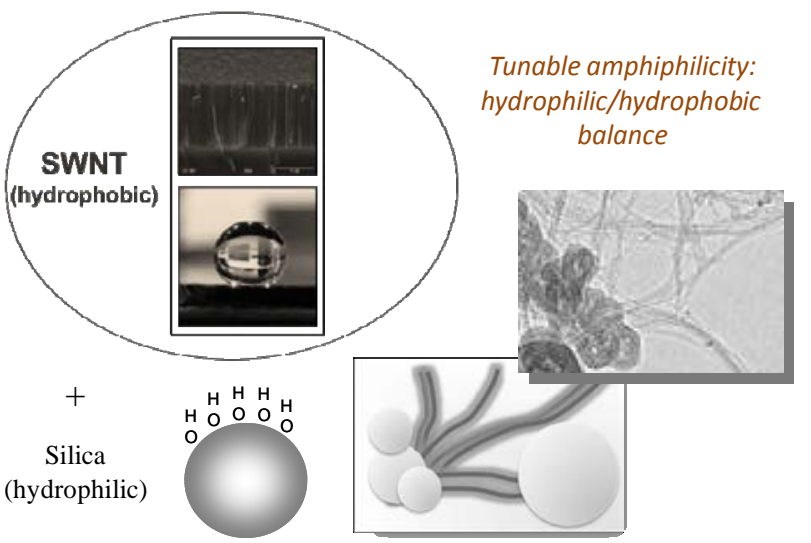

Fig. 2. Amphiphilic carbon nanotube/oxide particle nanohybrids, obtained by growing SWCNT on a Co-Mo catalyst supported on silica nanoparticles. The SWCNTs provide the hydrophobic component of the nanohybrid while silica provides the hydrophilic component.

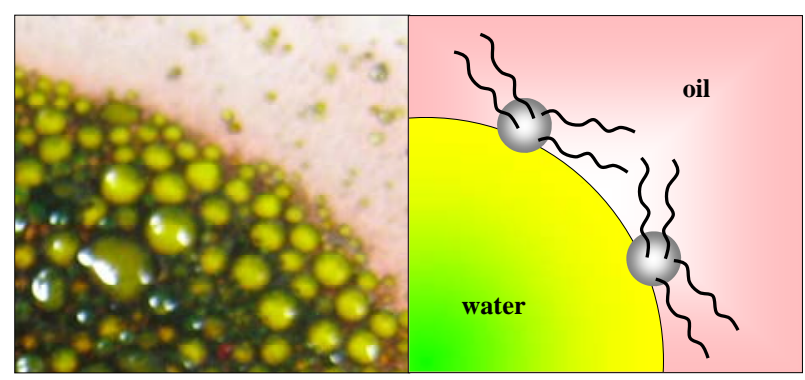

Fig. 3. Water-in-oil emulsion stabilized by SWCNT/silica nanohybrids. Water-soluble dye (green color, fluorescent Na salt) and oil soluble dye (red color, Sudan III) were added to identify the type of the emulsion. After Ref. [32].

alization (time, temperature, and $\mathrm{HNO}_{3}$ concentration) it is possible to adjust the nature of the resulting emulsion. The extent of functionalization can be quantified by Raman spectroscopy, based on the $D / G$ band ratio since oxidation leads to a higher intensity of the D band at $\sim 1300 \mathrm{~cm}^{-1}$, indicating the generation of oxidized defects with increased hydrophilicity $[32,44]$.

An increased hydrophilicity changes the curvature of the water/oil interface, forcing the oil phase to the inner part of the droplet as the nanohybrids are preferentially wetted by water. Then, for a given water/oil ratio in the mixture, one can invert the type of emulsion formed by simply increasing the hydrophilic/hydrophobic balance. It has been shown that the emulsion inversion can be predicted by the extent of CNT functionalization, as measured by the $\mathrm{D} / \mathrm{G}$ ratio [32].

A comparison of nanohybrids from different carbon materials was made in 2010 to test the effect of structural and chemical properties on the ability of nanohybrids to act as stabilizers of emulsions and anchoring supports for catalytic species [44]. The different nanohybrids are illustrated in Fig. 4 and included
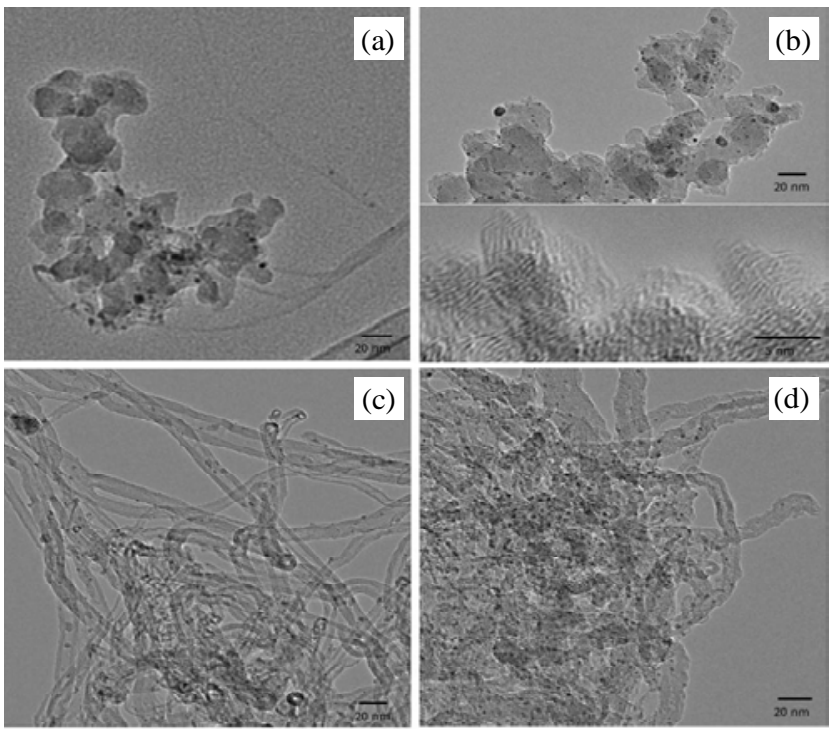

Fig. 4. HRTEM images of the catalysts composed of $5 \mathrm{wt} \% \mathrm{Pd}$ supported on different nanohybrids. (a) SWCNT-SiO 2 ; (b) "Onion-like" carbon- $\mathrm{SiO}_{2}$ (top: $\mathrm{Pd}$ on the nanohybrid; bottom: closer view of the "onion-like" carbon in the nanohybrid); (c) MWCNT- $\mathrm{Al}_{2} \mathrm{O}_{3}$; (d) Oxidized MWCNT- $\mathrm{Al}_{2} \mathrm{O}_{3}$. After Ref. [44]. 
"onion-like" carbon, SWCNTs and MWCNTs fused to silica or alumina particles. The amphiphilic character of these nanohybrids made them effective stabilizers of emulsions, comprising small droplets of less than $40 \mu \mathrm{m}$, as well as effective anchors for $\mathrm{Pd}$ and $\mathrm{Cu}$ nanoparticles to catalyze reactions at the aqueous/organic interface. Several reactions were tested in the emulsions, including hydrogenation of phenanthrene, glutaraldehyde and benzaldehyde, as well as oxidation of tetralin. Comparison of the maximum conversions achieved in emulsions as opposed to the single phase, together with much better control of selectivity in the two-phase system showed the benefits of using these nanohybrid catalysts.

As shown in Table 1, the different carbon nanomaterials exhibited a large range of defect densities, as assessed by Raman spectroscopy [44]. It can be seen that the $D / G$ ratio ranged from 0.09 for the SWCNT to 2.7 for the oxidized MWCNT. A direct correspondence between this density of defect and the metal dispersion (as measured by metal cluster size) was observed. That is, the smallest Pd particle size was obtained over the oxidized MWCNT, which had the higher density of oxidative defects, while the carbon surface with the least defects (pristine SWCNT) resulted in much larger Pd particles. Then, it is clear, that one of the secondary, but yet very important effects of oxidative functionalization is that, by changing the extent of surface functionalization, one can tailor the anchoring ability of the carbon materials to maximize the dispersion and stability of catalytic species, such as metal or oxide nanoclusters. It is reasonable to state that surface functional groups on carbon nanotubes result in a stronger interaction with these nanoclusters, leading to higher and more stable dispersions [45]. There are many examples in the literature that prove this concept. For instance, surface chemical modifications of CNTs by wet-chemical synthesis methods have been found to be highly effective in stabilizing noble metal [46] and oxide [47] nanoparticles. A recent theoretical/experimental combined study [48], has demonstrated that the dispersion of $\mathrm{Pd}$ nanoclusters on SWCNT can be greatly enhanced by creating defects on the nanotube walls, which lead to a stronger metal-support interaction. In that study, theoretical ONIOM (DFT:MM) calculations showed that the binding energy of Pd was significantly higher when the SWCNT surface was oxygen-functionalized than when it was a pristine SWCNT surface. The experimental measurements were consistent with the theoretical predictions. That is, TEM clearly showed that the functionalized SWCNT surface was much more effective than the pristine surface in anchoring Pd nanoclusters created by vapor deposition [48].

\section{Table 1}

G and D Raman band intensity ratio for various carbon nanohybrids compared to sizes of Pd particles stabilized over the corresponding nanohybrid. After Ref. [44].

\begin{tabular}{lccc}
\hline $\begin{array}{l}\text { Carbon } \\
\text { nanohybrid }\end{array}$ & $\begin{array}{c}\text { Raman } \mathrm{D} / \mathrm{G} \\
\text { ratio }\end{array}$ & $\begin{array}{c}\text { Defect } \\
\text { density }\end{array}$ & $\begin{array}{c}\text { Pd particle } \\
\text { size }(\mathrm{nm})\end{array}$ \\
\hline $\mathrm{SWCNT} / \mathrm{SiO}_{2}$ & 0.09 & very low & 4.9 \\
"Onion" carbon $/ \mathrm{SiO}_{2}$ & 1.1 & low & 4.6 \\
$\mathrm{MWCNT} / \mathrm{Al}_{2} \mathrm{O}_{3}$ & 2.3 & high & 3.0 \\
Oxid-MWCNT $/ \mathrm{Al}_{2} \mathrm{O}_{3}$ & 2.7 & very high & 2.1 \\
\hline
\end{tabular}

\section{Phase selectivity controlled by reactant solubility in biphasic liquid systems}

For reactions conducted in biphasic liquid/liquid systems, one can define "phase selectivity" as the contribution to overall conversion that occurs at each side of the liquid/liquid interface [49]. One can imagine several cases in which phase selectivity might be desirable. For example, in biofuel refining it might be desirable to combine the dehydration of sugars catalyzed by acid-base catalysts with the metal-catalyzed hydrogenation of the dehydrated product. Therefore, if a hydrogenation catalyst is placed in both phases and has no preferential orientation at the interface, the dehydration reaction will compete with hydrogenation, resulting in a decreased yield. By contrast, if a phase-selective hydrogenation catalyst is placed preferentially on the hydrophobic side, only the molecules that are stable in the organic phase undergo hydrogenation. More than forty years ago, Starks [50] observed that when two reactants, due to their low solubility in the other phase, are located in different phases the reaction among them is often inhibited by their inability to get together. To solve this problem, he proposed the use of small quantities of an agent that could transfer one reactant across the interface into the other phase, so that reaction could proceed. It was pointed out that since the phase-transfer agent was not consumed it could perform the transport function repeatedly. This concept was termed phasetransfer catalysis and has been widely used. The crucial notion to point out is that the role of the transfer agent is to facilitate the transport, which as described below, is the limiting factor when one of the reactants has a low solubility in a given phase.

\subsection{Experimental demonstration of phase selectivity}

The enhanced phase selectivity in an emulsion system when the solubilities of two reactants undergoing parallel reactions are very different in the two phases has been demonstrated in the following experiment. Water-soluble glutaraldehyde, $\mathrm{CH}_{2}\left(\mathrm{CH}_{2} \mathrm{CHO}\right)_{2}$ and oil-soluble benzaldehyde were simultaneously hydrogenated in an aqueous/organic emulsion over two different nanohybrids, which were used as stabilizers/catalysts [44]. One of the nanohybrids was SWCNT-SiO 2 and homogeneously impregnated with $5 \mathrm{wt} \% \mathrm{Pd}$ (i.e. Pd is on both sides of the nanohybrid); the other was a physical mixture of the metal-free nanohybrid (SWCNT-SiO 2 ) and silica-free $10 \mathrm{wt} \% \mathrm{Pd} / \mathrm{SWCNT}$ $(1: 1 \mathrm{w} / \mathrm{w})$. In the latter, the hydrophobic Pd/SWCNT remains in the oil side, but the strong tube-tube interaction keeps them at the water/oil interface, connected to the amphiphilic nanohybrids. In the former, Pd is distributed on both sides of the interface. The corresponding conversions of glutaraldehyde and benzaldehyde obtained over the two different catalysts after $3 \mathrm{~h}$ of reaction at $100{ }^{\circ} \mathrm{C}$ and $1.38 \mathrm{MPa}$ of $\mathrm{H}_{2}$, are compared in Table 2 [44]. The phase selectivity concept is clearly demonstrated. That is, the conversion of the organic-soluble benzaldehyde is very high when Pd is preferentially located in the oil phase and 55\% when $\mathrm{Pd}$ is present in both phases. The water-soluble glutaraldehyde exhibited the opposite behavior to that of benzaldehyde. Under the same conditions and in the 
Table 2

Simultaneous conversion of glutaraldehyde and benzaldehyde during hydrogenation over two different catalysts. After Ref. [44].

\begin{tabular}{lccr}
\hline \multirow{2}{*}{ Catalyst } & \multirow{2}{*}{ Pd present in } & \multicolumn{2}{c}{ Conversion (\%) } \\
\cline { 3 - 4 } & & Glutaraldehyde & Benzaldehyde \\
\hline $5 \mathrm{wt} \%$ Pd/SWCNT-SiO & both phases & 83 & 55 \\
$(1: 1)$ mixture of $\mathrm{SWCNT}_{-} \mathrm{SiO}_{2}$ nanohybrids $+10 \%$ Pd/purified SWCNT & only oil phase & 2.7 & 100 \\
\hline
\end{tabular}

Reaction conditions: Semi-batch reactor, catalyst $(30 \mathrm{mg})$, water/decalin $(30 \mathrm{~mL}, 1: 1 \mathrm{v} / \mathrm{v}), T=100{ }^{\circ} \mathrm{C}, \mathrm{H}_{2}(1.38 \mathrm{MPa}), t=3 \mathrm{~h}$.

simultaneous run the conversion was $83 \%$ when the catalyst contained $\mathrm{Pd}$ on both sides of the nanohybrids, but less than $3 \%$ when Pd was only deposited on the hydrophobic side [44].

\subsection{Thermodynamic, transport, and kinetic aspects of phase selectivity}

A word of caution should be inserted here concerning phase selectivity. It is well known that the driving force that determines chemical reaction is given by thermodynamic properties, such as chemical affinity, chemical potential or activity. Only in ideal reaction mixtures it is possible to express the reaction as a function of the concentration of the species present in the system [51]. For example, for the case of catalytic reactions in gas-liquid-solid systems, Madon et al. [52] pointed out that when the liquid and gas phases reached the thermodynamic phase equilibrium, the chemical potential of those phases became equal. Therefore, when the phases are equilibrated, the reaction rate should be independent of the location of the reactants and catalysts. Based on this premise, the concept of "phase selectivity" mentioned above would be unattainable. However, this principle does not apply when the phases are not in thermodynamic phase equilibrium, due to mass transfer limitations.

Therefore, the clear example of phase selectivity shown above is certainly due to the limited rate of mass transfer of each of the two reactants in the opposite phase. In fact, the expression $J_{i}^{A}=-\frac{D C_{i}^{A}}{R T} \frac{\partial \mu_{i}}{\partial x}$, represents the rate of mass transfer $\left(\mathrm{J}^{A}\right)$ of a solute $i$ in a given phase A, in terms of the gradient of chemical potential $\mu_{i}$, diffusivity $D$, and solubility $C_{i} A$. It is then obvious that while a gradient of chemical potential may exist between the catalyst in one phase and the reactant in another, the rate will be greatly limited if the solubility of a given species is very low in one of the phases. For instance, having a negligible solubility in oil, the rate of mass transfer of glutaraldehyde in decalin is practically zero. Therefore, the phase equilibrium cannot be accomplished during reaction.

\section{Examples of applications of catalytic reactions conducted in biphasic aqueous/organic emulsions stabilized by carbon nanohybrids}

The use of nanohybrids in biphasic aqueous/organic emulsions can have a number of interesting applications in several areas. The use of emulsions in reaction not only has resulted in expected enhanced mass transfer rates due to a larger interfacial area, but also in beneficial changes that include selectivity to desired compounds, catalyst stability, and reduced deactiva- tion. It has also resulted in composite materials of improved electrical properties. The following is a brief description of the most interesting results.

\subsection{Condensation-hydrodeoxygenation tandem reactions}

The first example is in the area of bio-oil upgrading. Bio-oil is a complex mixture of oxygenated compounds with up to $\sim 30 \%-40 \%$ of water produced from the pyrolysis of lignocellulosic biomass. Depending on the cooling process used in the condensation of the pyrolysis vapors, the crude bio-oil can generate a biphasic system, in which molecules are distributed, between the two phases, depending on their solubility. It is desirable to conduct reactions at the water/oil interface that can both enhance the fuel value of the molecules and effect phase migration, based on solubility, avoiding fractionation by heating (i.e. distillation), which is known to negatively impact bio-oil.

The use of carbon nanohybrids has resulted in a novel tandem catalysis concept that presents a unique advantage in streamlining biomass refining, where the immiscibility and thermal instability of crude bio-oil greatly complicates purification procedures. In this case, the metal oxide in the nanohybrid (MgO) acts both, as the hydrophylic side of the emulsifier, and as a basic catalyst for condensation reactions in the aqueous phase. Accordingly, small oxygenates soluble in water, with low fuel value, condense via aldol-condensation, resulting in products, which are no longer water-soluble and therefore migrate to the organic phase.

On the hydrophobic side of the nanohybrid, a Pd catalyst was placed, which catalyzes hydrogenation and hydrodeoxygenation. The concept of tandem catalysis is nicely illustrated in Fig. 5 [53]. In this particular example, acetone and furfural partition preferentially into the water droplets of the water-in-oil emulsion. The hydrophilic MgO particles face this side of the emulsion, and even under stirring they remained wetted by the aqueous liquid. Molecular dynamics studies have shown that even when a Janus particle is dragged into the oil phase, the hydrophilic part remains covered by a film of water. As a result, the oxide is in permanent contact with the aqueous phase, and it can effectively catalyze the condensation of the two oxygenates. The resulting condensation products: 4-(2-furyl)-3-buten2-one $\left(\mathrm{C}_{8}\right), 2$-furancarboxylic 2-furanmethyl ester $\left(\mathrm{C}_{10}\right)$ and 1,5-bis-(2-furanyl)-1,4-pentadien-3-one $\left(\mathrm{C}_{13}\right)$ have much lower solubilities in water and migrate out to the oil phase. With Pd selectively placed on the hydrophobic CNT, hydrogenation selectively occurs in the oil phase. As mentioned above, while in phase equilibrium the chemical potential of the light oxygenates in the oil phase should be the same as that in the water 


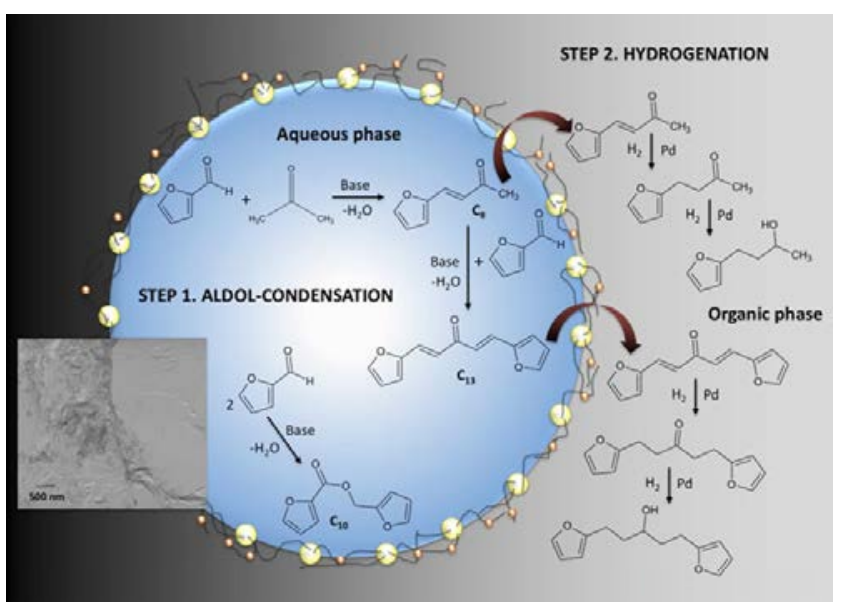

Fig. 5. Schematic description of the aldol condensation-hydrogenation tandem catalysis of furfural and acetone, conducted in an emulsion over a bifunctional nanohybrid catalyst. After Ref. [53].

phase, under reaction, their conversion quickly becomes masstransfer limited due to their low solubility in oil, and consequently, hydrogenation of furfural and acetone is very low. By contrast, as shown in Table 3, the larger condensation products are readily hydrogenated [53].

\subsection{Fischer-Tropsch synthesis in emulsions}

Conducting the FTS reaction in emulsion exhibits some attractive advantages [54]. One of them is the spontaneous product separation, simply based on solubility differences. A second important advantage is a higher catalyst tolerance against the presence of water-soluble impurities. In the specific case of FTS incorporated in biomass gasification processes, some of the common impurities are strong catalyst poisons (e.g. acids, ammonia and other N-compounds). With oil-in-water emulsions, the impurities can be continuously removed from the aqueous phase, minimizing the exposure of the catalyst to these poisons. Perhaps, the most important advantage is the enhanced activity and selectivity to desirable products $\left(\mathrm{C}_{5+}\right)$ recently described by Shi et al. [55]. In that study, the FTS was conducted over Ru supported on carbon nanotube-metal oxide nanohybrids at $200{ }^{\circ} \mathrm{C}$ in a batch reactor with $\mathrm{H}_{2} / \mathrm{CO}$ at a molar ratio around $2.0-3.5$, and at a total pressure of $4136.85 \mathrm{kPa}$. The most striking effects observed when using the biphasic mixture instead of a single solvent were an enhanced FTS activity due to the presence of condensed aqueous phase a re-

Table 3

Product distribution condensation/hydrogenation tandem reaction of furfural and acetone in a biphasic emulsion system. From Ref. [53].

\begin{tabular}{|c|c|c|c|c|c|c|c|c|c|c|c|c|}
\hline \multirow{2}{*}{ mmole Compound } & \multicolumn{4}{|c|}{ Feed } & \multicolumn{4}{|c|}{ Aldol condensation } & \multicolumn{4}{|c|}{ Hydrogenation } \\
\hline & Aqueous & Organic & Heavy & Total & Aqueous & Organic & Heavy & Total & Aqueous & Organic & Heavy & Total \\
\hline & 12.2 & 2.3 & 6.0 & 20.5 & 8.0 & 0.6 & 1.6 & 10.2 & 3.3 & 0.2 & 0.7 & 4.2 \\
\hline & 13.7 & 6.1 & 80.4 & 100.2 & 34.8 & 7.1 & 31.7 & 73.6 & 15.1 & 3.9 & 27.0 & 46.0 \\
\hline & - & - & - & - & 0.9 & - & 0.2 & 1.1 & 2.4 & 0.6 & 2.5 & 5.5 \\
\hline & - & - & - & - & 1.0 & 1.1 & 5.4 & 7.5 & - & 0.8 & 3.7 & 4.5 \\
\hline & - & - & - & - & - & - & - & - & - & 2.5 & 0.4 & 2.9 \\
\hline & - & - & - & - & - & - & - & - & 0.4 & - & 0.1 & 0.5 \\
\hline & - & - & - & - & 1.3 & - & 0.1 & 1.5 & 2.5 & - & 0.5 & 3.0 \\
\hline & - & - & - & - & 0.9 & - & 0.2 & 1.1 & 1.1 & 0.0 & 0.0 & 1.1 \\
\hline & - & - & - & - & - & - & - & - & 2.0 & - & 2.0 & 2.2 \\
\hline & - & - & - & - & - & 2.8 & 0.2 & 3.0 & 0.0 & 2.2 & 0.0 & 2.2 \\
\hline & - & - & - & - & - & - & - & - & - & 3.1 & 0.3 & 3.4 \\
\hline & - & - & - & - & - & - & - & - & 0.2 & - & 0.2 & 0.4 \\
\hline Carbon balance & $100.0 \%$ & & & & $92.8 \%$ & & & & $83.4 \%$ & & & \\
\hline
\end{tabular}

Catalysts: SWCNT/MgO for aldol condensation, and a physical mixture (wt. ratio 2:1) of SWCNT/MgO nanohybrids and $10 \mathrm{wt} \% \mathrm{Pd} / \mathrm{purified} \mathrm{SWCNT}$ for hydrogenation. Reaction conditions were $10 \mathrm{~h}$ at $80^{\circ} \mathrm{C}$ in $300 \mathrm{psi}$ of $\mathrm{He}$ for the aldol condensation reaction and then $17 \mathrm{~h}$ at $100{ }^{\circ} \mathrm{C}$ in $300 \mathrm{psi}$ of $\mathrm{H}_{2}$ for the hydrogenation reaction.

Feed: total molar concentration (furfural + acetone) of $1.8 \mathrm{~mol} / \mathrm{L}$; furfural/acetone molar ratio of 5:1. 
duced formation of methane and an enhanced $\mathrm{C}_{5+}$ selectivity, as summarized in Table 4. First, the initial activity enhancement in the presence of water has been recently explained by theoretical calculations, which show that the energy barrier for CO dissociation is much lower when it is assisted by surface $H[56,57]$. It is believed that water can enhance the rate of the kinetically-relevant $\mathrm{CO}$ activation by favoring the formation of $\mathrm{COH}^{*}$ via a $\mathrm{H}$-shuttling mechanism, in which $\mathrm{H}^{*}$ is transferred to a nearby $\mathrm{H}_{2} \mathrm{O}$ molecule to form a short-lived $\mathrm{H}_{3} \mathrm{O}^{\delta+}$ intermediate that protonates the $\mathrm{O}$ of $\mathrm{CO}^{*}$ [58]. The $\mathrm{COH}^{*}$ thus formed undergoes $\mathrm{H}^{\delta}$ - addition to the $\mathrm{C}$, forming an ${ }^{*} \mathrm{HCOH}^{*}$ intermediate and dissociates with nearby $\mathrm{H}_{2} \mathrm{O}$, which acts as a " $\mathrm{H}$-shuttling mediator" in the kinetically-relevant step. This mediating effect of water can also be responsible of the observed $\mathrm{C}_{5+}$ selectivity enhancement over $\mathrm{Ru}$ and Co catalysts when water is added [58].

Furthermore, the rate of deactivation in emulsion was found to be much lower than in the single-phase aqueous medium [55]. The improved characteristics of reaction in emulsion compared to single-phase aqueous medium were ascribed to the different abilities of the two media. In single-phase water, the solvent does not efficiently remove the hydrocarbon products; so, they tend to accumulate on the catalyst surface, causing deactivation. In the emulsion medium, the hydrocarbon products can more readily desorb from the catalyst surface into the oil due to their higher solubility in the oil phase. As a result, the surface can be kept free and available for continued chain growth. Therefore, contrary to the case of single-phase water, the emulsion can preserve active sites.

\subsection{Emulsion polymerization for production of conductive polymer composites}

The application of the nanohybrid-stabilized emulsions was further expanded to create composites of improved electrical properties. The high intrinsic conductivity of SWCNT is well known and there have been numerous studies that have investigated their use as conductive fillers of polymeric materials [59]. In general, it is difficult to obtain a good dispersion of the nanotubes and it is typically necessary to use higher concentrations than those theoretically predicted to reach the electrical percolation threshold [60]. By conducting the polymerization in oil-in-water emulsions stabilized by nanohybrids, it is possible to make conductive materials with extremely low amounts of SWCNT since they remain at the external area of the organic phase $[61,62]$. When polymerization is complete, each polymer

\section{Table 4}

Product distribution obtained at a conversion $\approx 65 \%$ in decalin, emulsion and water. From Ref. [55].

\begin{tabular}{lcccrc}
\hline \multirow{2}{*}{ Medium } & \multicolumn{4}{c}{ Product distribution (\%) } & Activity \\
\cline { 2 - 5 } & $\mathrm{C}_{1}-\mathrm{C}_{4}$ & $\mathrm{C}_{5}-\mathrm{C}_{12}$ & $\mathrm{C}_{13}-\mathrm{C}_{20}$ & $\mathrm{C}_{21+}$ & TOF \\
\hline Emulsion & 10.5 & 55.1 & 21.1 & 13.3 & 39 \\
Single decalin & 46.9 & 41.6 & 9.1 & 2.4 & 25 \\
Single water & 32.9 & 35.8 & 19.7 & 11.6 & 40 \\
\hline
\end{tabular}

All reactions were conducted with $150 \mathrm{mg}$ of catalyst at the same conditions of $4136.85 \mathrm{kPa}$ initial syngas $\left(\mathrm{H}_{2} / \mathrm{CO}=3.5\right)$ and $473.15 \mathrm{~K}$. particle formed from each organic droplet is covered by the nanohybrids, which form a slender network of conductive SWCNT. The polymerization of styrene was conducted in the oil-in-water emulsion stabilized by $\mathrm{SWCNT} / \mathrm{SiO}_{2}$ nanohybrids shown in Fig. 6(a). This emulsion was mixed with $\mathrm{AlCl}_{3} /$ hexadecane and $0.05 \mathrm{~g}$ of the oil-soluble initiator azo-bis-isobutyronitrile (AIBN) dissolved in $1.5 \mathrm{~mL}$ of pure ethanol. As shown in Fig. 6(b) and (c), the polymer particles retained about the same size and shape as the original emulsion droplets, indicating that the polymerization initiated by the oil-soluble AIBN occurred independently in each of the organic droplets, leaving the stabilizer nanohybrids on the external surface, thus forming an electrically conductive thin coating, as illustrated in Fig. 6(d).

To demonstrate the superior properties of this composite, a pellet was prepared by hot pressing the resulting dry composite (see Fig. 7). When cut and polished, the surface of the composite showed a characteristic cellular structure, in which each of the polystyrene particles are homogeneously coated with nanohybrids. A similar cellular structure has also been prepared by simply mixing SWCNT with powder polystyrene [63], however, in the emulsion method described here the resulting polymer particle size is almost one thousand times smaller. In addition to SEM, the composite samples were investigated by energy dispersive X-ray analysis (EDX). While the SWCNT cannot be independently detected by following the $\mathrm{C}$ signal, they are solidly connected to the $\mathrm{SiO}_{2}$ particles, so by following the Si signal, it is possible to determine the distribution of the nanohybrids. Therefore, as demonstrated by the EDX measurements of $C$ and Si shown in Fig. 7(c) and (d), the nanohybrids (containing $\mathrm{Si}$ ) are selectively positioned over the external surface of the polymer particles, forming a very thin layer.

After preparation, the volume electrical conductivity of hotpressed samples was measured by the four-point method. Composite disks of $\sim 1$ inch radius and $1.5 \mathrm{~mm}$ thickness were
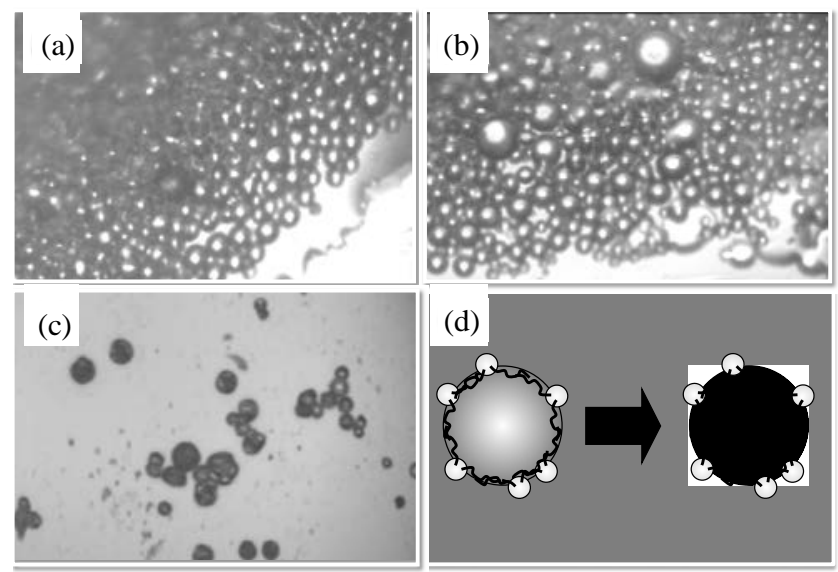

Fig. 6. Emulsion polymerization of styrene. (a) Original oil-in-water stabilized by $\mathrm{SWCNT} / \mathrm{SiO}_{2}$ nanohybrids; (b) Emulsion added to a mixture of $\mathrm{AlCl}_{3} /$ hexadecane and $0.05 \mathrm{~g}$ of azo-bis-isobutyronitrile (AIBN) dissolved in $1.5 \mathrm{~mL}$ of pure ethanol; (c) Polymer particles retain the same size and shape as the original emulsion droplets; (d) Schematic showing conversion of the oil-in-water emulsion to polymer particles covered by $\mathrm{SWCNT} / \mathrm{SiO}_{2}$ nanohybrids. After Refs. [61,62]. 


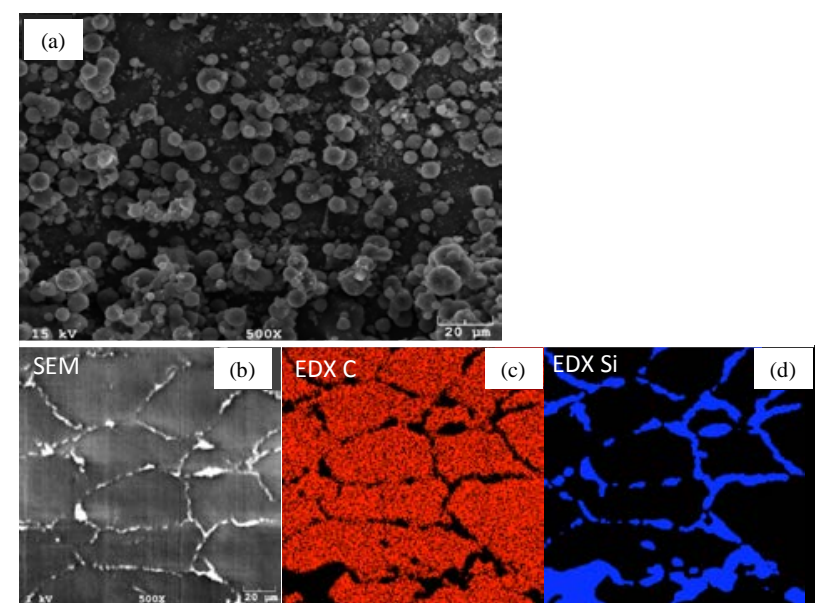

Fig. 7. (a) SEM image of the polymer-nanohybrid composite particles produced in the nanohybrid-stabilized emulsion. (b) SEM image of the resulting composite prepared by hot pressing the particles shown in (a); Carbon (c) and Silicon (d) EDX mapping of the corresponding image, clearly showing that the $\mathrm{SiO}_{2}$-containing nanohybrids are concentrated along the intergranular regions of the composite. After Refs. $[61,62]$.

tested at room temperature following ASTM D 257 for insulating materials (i.e., resistivities $>1 \times 10^{7} \mathrm{ohm}-\mathrm{cm}$ ) using a Keithley 6105 Ring Conductivity Adapter regulated by a power supply and a 610C multi-range electrometer. Four-point probe Jandel HM21 conductivity equipment was used to measure voltage for moderately conductive materials (i.e., resistivities < $\left.1 \times 10^{7} \mathrm{ohm}-\mathrm{cm}\right)$. The results are summarized in Fig. 8. It is clear that the electrical properties of this material are indeed remarkable. When compared to samples prepared by conventional mixing of polymer and SWCNT in an organic solvent, those prepared by the nanohybrid-stabilized emulsion method displayed electrical percolation thresholds at more than an order of magnitude lower carbon loadings.

\section{Conclusions}

Carbon nanohybrids are effective amphiphilic particles that

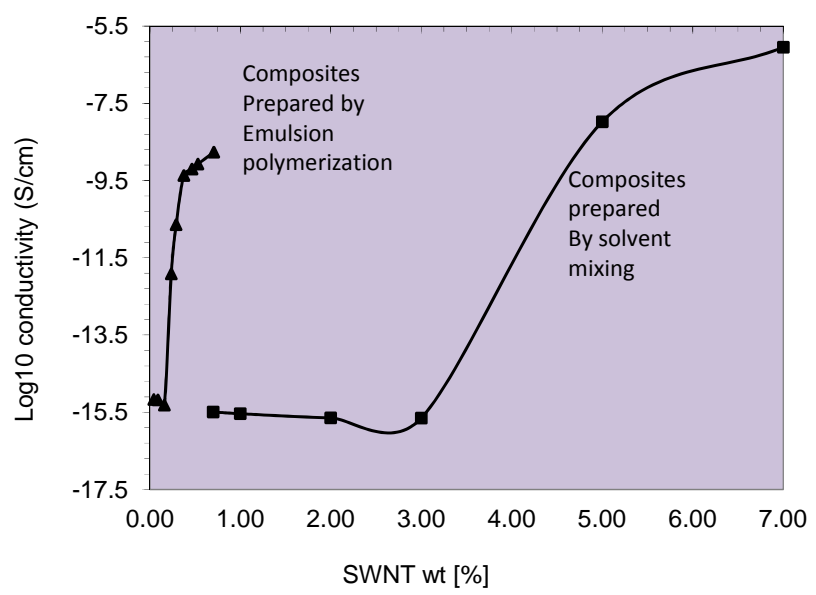

Fig. 8. Electrical conductivity measurements of the SWCNT/polystyrene composites prepared by emulsion polymerization stabilized by nanohybrids and conventional mixing of SWCNT and dissolved polystyrene. After Refs. [61,62].

can be used as recoverable and reusable catalysts for a number of interesting applications. In such systems, using an emulsion stabilized by fine solid particles offers significant advantages such as an increased interfacial area, enhanced mass transfer of molecules between the two phases, simplified reaction/separation process by using a recoverable solid catalyst instead of surfactant, and effective separation of products from the reaction mixture. An important point is that the separation is accomplished by different partitions in the water/oil media. Therefore, one can avoid heating the products, which in cases like oxygenated compounds may lead to decomposition or deterioration. These process improvements have been shown to have a major impact in the field of biomass conversion to fuels, Fischer-Tropsch synthesis, and synthesis of conductive polymeric composites. However, the overall concept may have impact on many other areas, in which the use of biphasic media is either unavoidable or beneficial, or when the products can be more conveniently separated by differences in solubility than by thermal fractionation.

\section{Graphical Abstract}

Chin. J. Catal., 2014, 35: 798-806 doi: 10.1016/S1872-2067(14)60119-4

\section{Carbon nanohybrids used as catalysts and emulsifiers for reactions in} biphasic aqueous/organic systems

Daniel E. Resasco*

University of Oklahoma, USA

Carbon nanohybrids can stabilize emulsions and serve as supports for species that can catalyze tandem reactions in biphasic aqueous/organic mixtures used in biomass conversion and Fischer-Tropsch synthesis, with significant advantages in activity, selectivity, and separations.

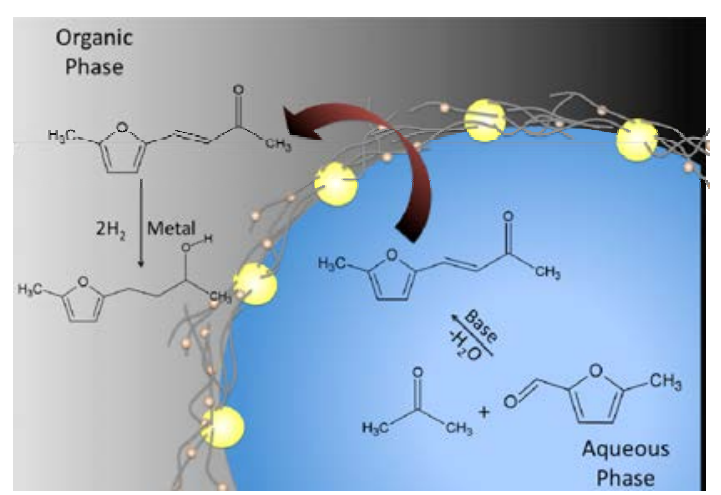




\section{References}

[1] Li N, Tompsett G A, Zhang T Y, Shi J A, Wyman C E, Huber G W. Green Chem, 2011, 13: 91

[2] Joo F. Acc Chem Res, 2002, 35: 738

[3] Wiebus E, Cornils B. In: Cole-Hamilton D J, Tooze R P eds. Catalysis Separation, Recovery and Recycling. Dordrecht, Netherlands: Springer, 2006. 105

[4] Schmitt T M. Analysis of Surfactants. 2nd Ed. New York: Marcel Dekker, Inc, 2001

[5] Crossley S, Faria J, Shen M, Resasco D E. Science, 2010, 327: 68

[6] Cole-Hamilton D J. Science, 2010, 327: 41

[7] Ungureanu S, Deleuze H, Sanchez C, Popa M I, Backov R. Chem Mater, 2008, 20: 6494

[8] Huber G W, Iborra S, Corma A. Chem Rev, 2006, 106: 4044

[9] Vispute T P, Zhang H Y, Sanna A, Xiao R, Huber G W. Science, 2010, 330: 1222

[10] Huber G W, Dumesic J A. Catal Today, 2006, 111: 119

[11] Roman-Leshkov Y, Barrett C J, Liu Z Y, Dumesic J A. Nature, 2007 447: 982

[12] Kunkes E L, Simonetti D A, West R M, Serrano-Ruiz J C, Gartner C A, Dumesic J A. Science, 2008, 322: 417

[13] Casanova O, Iborra S, Corma A.J Catal, 2010, 275: 236

[14] Gao J B, Wang S G, Jiang Z X, Lu H Y, Yang Y X, Jing F, Li C. J Mol Catal A, 2006, 258: 261

[15] Lü H Y, Gao J B, Jiang Z X, Jing F, Yang Y X, Wang G, Li C. J Catal, 2006, 239: 369

[16] Van Steen E, Claeys M. Chem Eng Technol, 2008, 31: 655

[17] Bridgwater A V. Therm Sci, 2004, 8(2): 21

[18] Binks B P, Fletcher P D I. Langmuir, 2001, 17: 4708

[19] Binks B P, Lumsdon S 0. Phys Chem Chem Phys, 1999, 1: 3007

[20] Hunter T N, Pugh R J, Franks G V, Jameson G J. Adv Colloid Interf Sci, 2008, 137: 57

[21] Arditty S, Whitby C P, Binks B P, Schmitt V, Leal-Calderon F. Eur Phys JE, 2003, 11: 273

[22] Aveyard R, Clint J H, Horozov T S. Phys Chem Chem Phys, 2003, 5: 2398

[23] Kralchevsky P A, Ivanov I B, Ananthapadmanabhan K P, Lips A. Langmuir, 2005, 21: 50

[24] Binks B P. Curr Opin Colloid Interface Sci, 2002, 7: 21

[25] Binks B P, Rodrigues J A. Angew Chem Int Ed, 2005, 44: 441

[26] Stadler R, Auschra C, Beckmann J, Krappe U, Voigt-Martin I, Leibler L. Macromolecules, 1995, 28: 3080

[27] Takei H, Shimizu N. Langmuir, 1997, 13: 1865

[28] Forster S, Antonietti M. Adv Mater, 1998, 10: 195

[29] Liu Y F, Abetz V, Muller A H E. Macromolecules, 2003, 36: 7894

[30] Vanakaras A G. Langmuir, 2006, 22: 88

[31] Cayre O, Paunov V N, Velev O D. Chem Commun, 2003: 2296

[32] Shen M, Resasco D E. Langmuir, 2009, 25: 10843

[33] Prasomsri T, Shi D C, Resasco D E. Chem Phys Lett, 2010, 497: 103

[34] Bancroft W D. J Phys Chem, 1913, 17: 501
[35] Morrison I D, Ross S. Colloidal Dispersions: Suspensions, Emulsions and Foams. New York: Wiley, 2002

[36] Pickering S U.J Chem Soc, 1907, 91: 2001

[37] Resasco D E, Alvarez W E, Pompeo F, Balzano L, Herrera J E, Kitiyanan B, Borgna A.J Nanopart Res, 2002, 4: 131

[38] Arthur D, Silvy R P, Wallis P, Tan Y Q, Rocha J D R, Resasco D, Praino R, Hurley W. MRS Bull, 2012, 37: 1297

[39] Chen J, Hamon M A, Hu H, Chen Y S, Rao A M, Eklund P C, Haddon R C. Science, 1998, 282: 95

[40] Tchoul M N, Ford W T, Lolli G, Resasco D E, Arepalli S. Chem Mater, 2007, 19: 5765

[41] Singh J P, Zhang X G, Li H L, Singh A, Singh R N. Int J Electrochem Sci, 2008, 3: 416

[42] Satishkumary B C, Voglz E M, Govindaraj A, Rao C N R. J Phys D, 1996, 29: 3173

[43] Bittencourt C, Felten A, Douhard B, Colomer J-F, Van Tendeloo G, Drube W, Ghijsen J, Pireaux J J. Surf Sci, 2007, 601: 2800

[44] Ruiz M P, Faria J, Shen M, Drexler S, Prasomsri T, Resasco D E. ChemSusChem, 2011, 4: 964

[45] Felten A, Ghijsen J J, Pireaux J J, Drube W, Johnson R L, Liang D, Hecq M, Van Jendeloo G, Bittencourt C. Micron, 2009, 40: 74

[46] Li W Z, Liang C H, Zhou W J, Qiu J S, Zhou Z H, Sun G Q, Xin Q.J Phys Chem B, 2003, 107: 6292

[47] Liu C C, Bolin T, Northrup P, Lee S, McEnally C, Kelleher P, Pfefferle L, Haller G L. Top Catal, 2014, 57: 693

[48] Shi D C. [PhD Dissertation]. Norman, OK: The University of Oklahoma, 2014

[49] Faria J, Ruiz M P, Resasco D E. Adv Synth Catal, 2010, 352: 2359

[50] Starks C M. J Am Chem Soc, 1971, 93: 195

[51] Madon R J, Iglesia E. J Mol Catal A, 2000, 163: 189

[52] Madon R J, O'Connell J P, Boudart M. AIChE J, 1978, 24: 904

[53] Zapata P A, Faria J, Pilar Ruiz M, Resasco D E. Top Catal, 2012, 55: 38

[54] Shi D C, Faria J A, Rownaghi A A, Huhnke R L, Resasco D E. Energy Fuels, 2013, 27: 6118

[55] Shi D C, Faria Albanese J A, Pham T N, Resasco D E. ACS Catal, 2014, DOI: $10.1021 / \operatorname{cs} 500040 \mathrm{n}$

[56] Ojeda M, Nabar R, Nilekar A U, Ishikawa A, Mavrikakis M, Iglesia E. J Catal, 2010, 272: 287

[57] Loveless B T, Buda C, Neurock M, Iglesia E. J Am Chem Soc, 2013, 135: 6107

[58] Hibbitts D D, Loveless B T, Neurock M, Iglesia E. Angew Chem Int Ed, 2013, 52: 12273

[59] Sandler J K W, Kirk J E, Kinloch I A, Shaffer M S P, Windle A H. Polymer, 2003, 44: 5893

[60] Pfeifer S, Park S H, Bandaru P R. J Appl Phys, 2010, 108: 024305/1

[61] Barraza H J, Balzano L, Pompeo F, Rueda O L, O'Rear E A, Resasco D E. US Patent 7153903 B2. 2006

[62] Shen M, Resasco D E. unpublished

[63] Mu M F, Walker A M, Torkelson J M, Winey K I. Polymer, 2008, 49: 1332 